Revisão

\title{
Transplante de medula óssea em leucemia mielóide aguda
}

Daniel G. Tabak

\begin{abstract}
O transplante de medula óssea constitui terapêutica eficaz no tratamento da leucemia mielóideaguda. Embora utilizado inicialmenteem pacientes em fases tardias da doença, os melhores resultados são documentados em pacientes submetidos ao procedimento en quanto em primeira remissão. Avanços no manu seio do paciente neutropênico permitem hojea utilização com maior segurança de regi mes quimioterápicos agressivos que resultam em uma sobrevida prolongada, semelhanteàquela observada pós regimes mieloablativos. Ainda existem dúvidas sobre quais os pacientes que deveriam ser submetidos às diferentes modalidades de intensificação. Os estu dos citogenéticos euma melhor definição das características biológicas de cada indivíduo permitirão uma melhor seleção de pacientes. 0 melhor controle da Doença do Enxerto contra Hospedeiro, o melhor manuseio das complicações infecciosas pós transplante, a utilização de regimes de condicionamento menos agressi vos ea maior disponibilidade de doadores não aparentados permite antever uma maior aplicabilidade do transplante de medula óssea alogênico no tratamento da Leucemia MielóideAguda, inclusiveem pacientesmaisidosos. O papel do transplante autólogo precisa ser melhor definido.
\end{abstract}

Rev.bras.hematol.hemoter., 2000, 22(1):55-62

Palavras-chave: leucemia mielóide aguda, transplante de medula, tratamento

\section{Introdução}

0 transplante de medula óssea (TMO) vem sendo utilizado no tratamento da leucemia mielóide aguda (LMA) há mais de 15 anos. Thomas e colaboradores descreveram 100 pacientes com leucemia aguda que falharam com o tratamento inicial e encontravam-se em vários estados de sua evolução. 0 relato incluía 54 pacientes com LMA. Seis pacientes permanecem livres de doença por um período de acompanhamento superior a 11 anos (1).

0 melhor manuseio das complicações permitiu a utilização progressiva do TMO em estádios iniciais da doença. Avanços ocorreram em múltiplos aspectos no cuidado do paciente transplantado. Estes incluem o uso de irradiação corporal fracionada como uma alternativa a uma única fração, e a agentes mais eficazes na profilaxia de Doença Enxerto contra o Hospedeiro (DECH) e terapia eficaz para reativação de infecções pelo citomegalovírus (CMV), determinando uma redução na mortalidade associada ao TMO (2).

\section{Transplante a logênico de medula óssea em LMA em 1a Remissão}

Cerca de $70 \%$ dos pacientes adultos com LMA entram em remissão completa após a quimioterapia convencional utilizando citarabina

Centro de Transplante de Medula Óssea

Instituto Nacional de Câncer.

Endereço para correspondência: Praça da Cruz Vermelha, 23 - 70 andar. Cep.: 20230-130. Rio de Janeiro. RJ 
e um antracíclico. Entretanto, apenas 20-30\% apresentam uma remissão duradoura com a consolidação convencional. Um estudo do CALGB (3) demonstrou uma sobrevida superior em pacientes adultos com idade inferior a 60 anos que receberam consolidação com vários ciclos de citarabina. Porém estes dados ainda não foram reproduzidos. Mais recentemente foi descrito uma resposta mais favorável em pacientes que apresentavam a $\mathrm{t}(8 ; 21)$ ao diagnóstico (4).

Vários estudos se sucederam com o objetivo de avaliar a eficácia do TMO como terapia de consolidação na LMA em 1 à remissão. A sobrevida livre de doença em vários destes estudos variou entre 40 e $60 \%$ com taxas de recaída na ordem de $20 \%$ (Figura 1) (5). Resultados semelhantes também foram observados no Brasil (Figura 2) (6). A DECH continua afetando significativamente a sobrevida. Foram realizadas várias tentativas no sentido de modificar este equilíbrio ( $D E C H \quad x$ recidiva), pois uma redução na taxa de $\mathrm{DECH}$ frequentemente resulta em um aumento da taxa de recaídas. Este fenômeno pode ser observado com a associação de ciclosporina e metotrexate e com a depleção de células $T$ do enxerto. As duas estratégias diminuem a incidência de DECH e estão associados com uma maior incidência de recidivas pós transplante. Mais recentemente, métodos seletivos de depleção $T$ vem sendo testados no sentido de preservar o efeito "graft

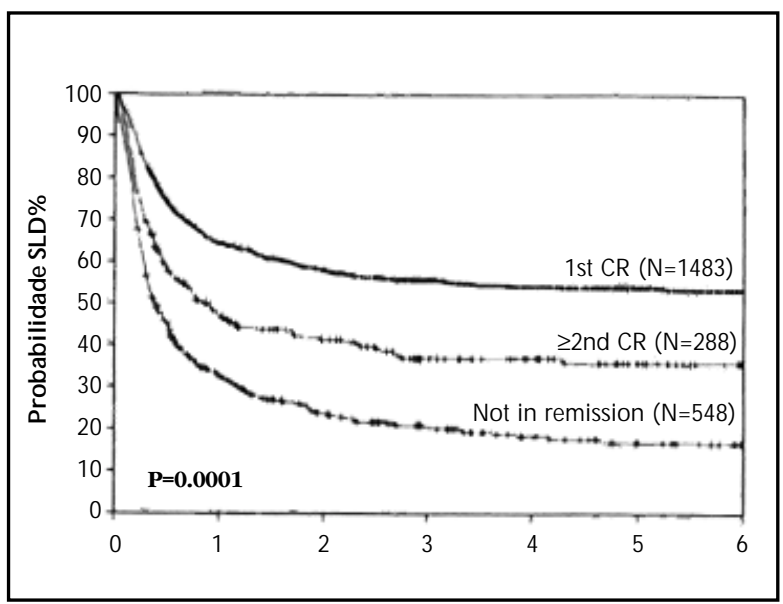

Figura 1. Transplante de Medula Óssea em Leucemia Mielóide Crônica Aguda Resultados do IBMTR versus leukemia", reduzindo as complicações associadas a DECH. Os estudos ainda são limitados e a análise prospectiva é necessária no sentido de verificar o impacto sobre a sobrevida global (7).

0 mesmo efeito pode ser demonstrado em um estudo publicado recentemente, no qual doses reduzidas de ciclosporina foram comparadas com o tratamento convencional na profilaxia da DECH em crianças submetidas a TMO para tratamento de leucemia aguda. Apesar de incluir um pequeno número de pacientes, este estudo prospectivo demonstrou uma menor taxa de recidiva nos pacientes que receberam a dose reduzida de CSA. Entretanto, foi também observada uma maior incidência de DECH agudo grau II-IV nas primeiras 3 semanas após o transplante (8).

Vários regimes preparatórios vem sendo utilizados para o tratamento destes pacientes. A maioria dos estudos foi incapaz de demonstrar a superioridade de qualquer um deles. Entretanto, Blaise e colaboradores (9) publicaram um estudo prospectivo comparando a associação de bussulfan e ciclofosfamida (BUCY) com a irradiação corporal total em 101 pacientes. Foi observada uma maior taxa de recidiva para os pacientes que receberam BUCY, resultados que refletem uma menor sobrevida livre de eventos avaliada em 4 anos. Estes dados são contrários a estudos retrospectivos anteriores e podem estar relacionados a uma menor absorção intestinal

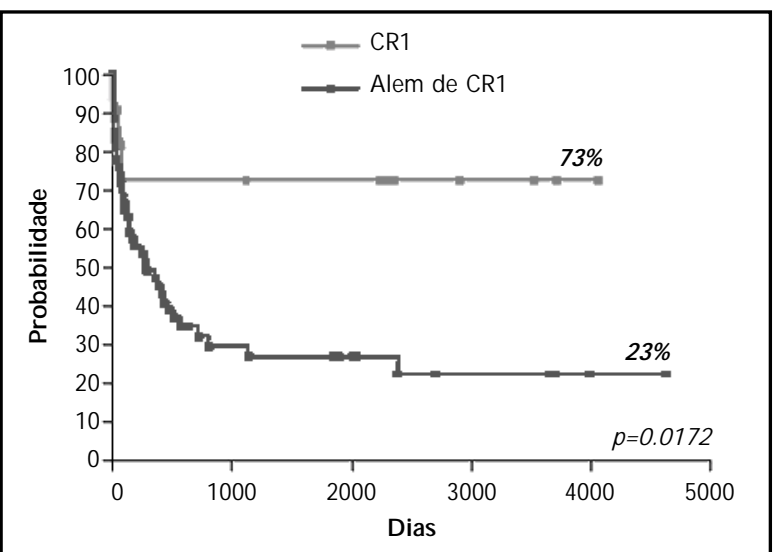

Figura 2. Transplante de Medula Óssea em Leu cemia Mielóide Crônica Aguda: Resultados do Centro de Transplante de Medula Óssea (CEMO/INCA) (9) 
do bussulfan. Ainda com o objetivo de reduzir as taxas de recidiva pesquisadores em Seattle (10) comparam dois regimes utilizando doses diferentes de irradiação corporal total. Pacientes que receberam uma dose total de 15,756 y apresentaram uma menor taxa de recidiva que aqueles que receberam $12.0 \mathrm{~Gy}$. Este fenômeno não resultou em uma melhor sobrevida, pois 0 regime mais intenso também foi associado com uma maior toxicidade.

Alterações citogenéticas constituem um importante fator prognóstico na leucemia mielóide aguda. Algumas anomalias estão associadas com uma evolução mais favorável, incluindo $t(8 ; 21), t(15 ; 17)$ e inv16. Alguns estudos descrevem uma sobrevida livre de recidivas em até $84 \%$ dos pacientes, tratados com altas doses de ARA-C. 0 mesmo fenômeno pode ser observado em pacientes submetidos ao transplante alogênico. Pacientes com um cariótipo normal ou ausência do cromossoma $Y$, apresentam um prognóstico intermediário. Todas as demais anomalias citogenéticas determinam uma evolução menos positiva (11).

Desta forma os resultados do transplante dependem do equilíbrio entre fatores definidos antes da infusão das células doadoras, como o estádio e a biologia da doença e a idade do paciente. Fatores de co-morbidade associados ao regime de condicionamento e condições que influenciam o curso pós TMO - DECH, infecções bacterianas, fúngicas e virais - também exercem um papel crítico.

\section{Qual o momento ideal para a utilização do transplante alogênico em LMA?}

Embora as taxas de recidivas sejam menores quando 0 transplante é realizado em 1 à remissão, esta estratégia acaba expondo $20-40 \%$ de pacientes já curados pela quimioterapia à toxicidade do TMO. Como o transplante também constitui uma terapia eficaz em pacientes que recidivam, uma alternativa consiste em retardar o transplante até que seja observada uma recaída. Uma análise retrospectiva conduzida pelo International Bone Marrow Transplant Registry (IBMTR), os resultados do TMO alogênico em pacientes adultos com idade inferior a 50 anos com o diagnóstico de LMA em $2^{\text {a }}$ remissão foi comparado com os resultados de quimioterapia. Para pacientes com idade inferior a 30 anos e com uma duração de $1^{\text {a }}$ remissão superior a 1 ano, os resultados com 0 TMO alogênico são claramente superiores em termos de sobrevida livre de eventos em 3 anos (41\% x 17\%). Para pacientes com idade superior a 30 anos e com uma duração de remissão superior a 12 meses, os resultados foram superiores porém menos significativos (18\% x 7\%) (12).

Para os pacientes não transplantados em primeira remissão e que apresentam uma recidiva, existem dúvidas sobre a necessidade de quimioterapia pré TMO com o objetivo de induzir uma segunda remissão. Resultados iniciais sugerem que os resultados obtidos para pacientes em primeira recidiva não são inferiores aqueles obtidos em segunda remissão (13). Esta abordagem entretanto, necessita que o paciente seja rapidamente encaminhado ao centro de transplante, minimizando as complicações infecciosas frequentemente associadas a uma reindução.

0 transplante alogênico também pode ser considerado para aqueles pacientes que apresentam falha na indução de remissão. Estudos recentes confirmam que cerca de $20 \%$ destes pacientes podem ser resgatados com esta estratégia terapêutica (14).

\section{Transplante Autólogo de Medula Óssea em LMA}

Para pacientes que não possuem um doador compatível, o transplante autólogo de células hematopoiéticas constitui uma alternativa terapêutica. Os primeiros estudos não randomizados surgiram em 1980, com a idéia que o tratamento mieloablativo poderia ser utilizado de forma segura em pacientes com idade até 60 anos conforme a experiência nos transplantes singênicos (2). Esta estratégia poderia ser oferecida para um grande número de pacientes que entrassem em remissão. Apesar da preocupação com a reinfusão de células neoplásicas, modelos matemáticos na época sugeriam um significado menor para este problema. Estudos mais recentes utilizando a marcação genética de células do enxerto, entretanto, demonstram a importância de células residuais como fonte 
de recidivas. Este fenômeno mostrou-se importante mesmo quando estudos moleculares eram incapazes de caracterizar a presença de doença neoplásica residual (15). Existem dúvidas de que a utilização de precursores hematopoéticos de sangue periférico como fonte de células tronco estaria asso ciada a uma menor taxa de recidivas (16).

\section{TMO Autólogo - Estudos não randomizados em 1 a remissão}

Vários grupos reportaram séries de pacientes submetidos a transplante de medula óssea como consolidação da 1 à remissão. Estes pacientes receberam vários regimes quimioterápicos distintos e diferentes regimes de condicionamento. Os resultados, entretanto, foram muito semelhantes com uma sobrevida livre de doença de 45 a $50 \%$ em 5 anos. A mortalidade associada ao procedimento foi baixa com uma frequência pequena de doença associada a reativação do CMV. Um aspecto comum é a lenta recuperação plaquetária correlacionada habitualmente com os índices hematológicos por ocasião da coleta de células (17).

As recidivas em autotransplante ocorrem mais frequentemente nos primeiros 18 meses pós TMO. A análise do Registro Europeu demonstrou que o tempo de remissão pré TMO apresentava boa correlação com o risco de recidiva após o procedimento. Duas condições podem ser reconhecidas: quanto maior o tempo em remissão menor o risco de uma recidiva. 0 segundo aspecto é que os resultados podem ser otimizados se o transplante for precedido por terapia de consolidação, determinando uma citoredução máxima "in vivo" (18).

\section{TMO Autólogo - Estudos em 2a remissão}

Cerca de $50 \%$ dos pacientes submetidos a um transplante autólogo em 2 a remissão podem ser curados. Foi neste contexto que o tratamento "in vitro" de medula óssea com derivados de ciclofosfamida foi concebido. O valor do tratamento "in vitro" entretanto, ainda não pode ser confirmado em estudos prospectivos. Os principais fatores associados com a durabilidade das respostas parecem ser a idade do paciente, a duração da $1^{a}$ remissão e o cariótipo. Qual a conduta no paciente cuja medula óssea já foi criopreservada e apresenta uma recidiva? Cerca de $50 \%$ destes pacientes não atingem uma nova remissão, e mesmo quando ela ocorre, em um número significativo de pacientes, o TMO tornase contraindicado devido às implicações do tratamento quimioterápico. Quando a recidiva ocorre tardiamente, em paciente jovem que apresenta um cariótipo favorável, eles apresentam uma grande probabilidade de entrar em remissão. Nas recidivas precoces os resultados geralmente são desastrosos com o tratamento quimioterápico convencional. Alguns estudos sugerem, entretanto, que a sobrevida após um autotransplante pode atingir 20\% em 4 anos, em um grupo selecionado de pacientes (18).

\section{Tratamento "In Vitro" - Necessário?}

Apesar de antigo o debate continua sobre a necessidade do tratamento "in vitro" da medula óssea e como medir os benefícios associados a ele. A análise do registro europeu (20) demonstrou uma redução de risco de recidiva em pacientes submetidos a irradiação corporal total e que receberam medula óssea tratada. Dois subgrupos parecem ser preferencialmente beneficiados: os pacientes nos quais a documentação de remissão ocorre após 40 dias e aqueles nos quais o transplante é realizado precocemente $(<6 \mathrm{~m})$. Nestes grupos, a taxa de recidiva foi reduzida de 58 para $37 \%$. Este risco de recidiva é inferior aquele observado em transplantes singênicos, sugerindo um efeito imunológico adicional pela manipulação "in vitro". Existem evidências preliminares que a atividade "natural-killer" possa ser amplificada no período pós transplante em pacientes que recebem medula óssea tratada "in vitro" (19).

\section{Quando deve ser realizado autotransplante em LMA?}

Existem autores que recomendam a sua utilização em primeira remissão, após uma consolidação intensiva, com o objetivo de reduzir "in vivo" a massa tumoral. Alternativamente, 0 autoenxerto poderia ser reservado para pacientes que falharam ao tratamento inicial. Neste caso, células tronco colhidas na primeira remissão seriam utilizadas 
em caso do transplante ser realizado em recidiva ou em segunda remissão.

Devido à seleção de pacientes seria muito difícil concluir qual o papel real do transplante autólogo no tratamento de pacientes portadores de LMA. 0 tempo de realização do autotransplante constitui um aspecto de muita importância pois pacientes em remissão por 4 a 6 meses apresentam 30 - 40\% de possibilidades de permanecer em remissão por um período de 5 anos, sem qualquer outro tratamento adicional (20).

\section{Estudos prospectivos em LMA: Transplante alogênico $x$ Transplante autólogo $x$ Quimioterapia intensiva (21)}

Considerando a evidência que a terapêutica de intensificação pós indução de remissão contribui para uma maior sobrevida livre de recidiva em pacientes em 1 a remissão, bem como o benefício potencial do efeito enxerto contra leucemia associado ao transplante alogênico, vários estudos procuraram comparar os benefícios da consolidação com doses elevadas de citarabina, o transplante autólogo de medula óssea ou de células tronco periférico e o transplante alogênico de medula óssea. Como descrito anteriormente 0 autotransplante permite 0 escalonamento de doses mas não apresenta 0 efeito enxerto contra leucemia associado como o transplante alogênico e apresenta a complicação potencial da reinfusão de células leucêmicas ocultas. 0 transplante alogênico oferece 0 melhor potencial antileucêmico, mas é associado com o maior risco de mortalidade. Vários estudos foram realizados nos quais os pacientes jovens com um doador HLA idêntico são encaminhados para o transplante alogênico, enquanto os demais pacientes são randomizados para 0 autotransplante ou quimioterapia de consolidação. Zittoun e colaboradores conduziram o primeiro estudo através de uma colaboração do EORTC e o GIMEMA. Eles analisaram 168 pacientes em primeira remissão submetidos a transplante alogênico e randomizaram 126 pacientes a um segundo ciclo de consolidação com $16 \mathrm{~g} / \mathrm{m}^{2}$ de citarabina e daunorubicina e 128 pacientes ao transplante autólogo, condicionados com ciclofosfamida e irradiação corporal total.
Apenas $54 \%$ dos pacientes encaminhados para o transplante autólogo realmente receberam 0 tratamento. A análise de sobrevida livre de doença em 4 anos por "intenção de tratar" revelou resultados semelhantes para o transplante autólogo (50\%) e alogênico (55\%). A sobrevida global nos três grupos foi semelhante (59\%: alogênico; 56\%: autólogo; e 46\% quimioterapia). Embora as recidivas ocorressem com maior freqüência pós transplante autólogo, a mortalidade foi mais frequentemente associada ao transplante alogênico. No estudo conduzido pelo ECOG/SWOG/CALGB, embora o regime de consolidação fosse distinto, os resultados foram semelhantes. Foi observada uma discreta superioridade na sobrevida livre de doença em 4 anos para os pacientes encaminhados para 0 transplante alogênico quando comparada ao autotransplante ou a quimioterapia convencional porém, sem significância estatística (43\% x $34 \% \times 34 \%)$. o estudo realizado por Harousseau e cols para o GOELAM mostrou os mesmos resultados. 0 ensaio clínico realizado pelo MRC e publicado por Burnett e cols. incluiu quase 2000 pacientes e após uma consolidação intensiva, os pacientes não encaminhados para o autotransplante, permaneceram apenas em observação. Neste estudo, o transplante autólogo reduziu significativamente o risco de recidivas com um efeito demonstrável na sobrevida global e sobrevida livre de eventos em 7 anos (57\% x 45\%). Este estudo sugere que 0 efeito do autotransplante pode estar condicionado a uma consolidação intensiva anterior. 0 mesmo fenômeno, entretanto, não foi documentado em crianças. A análise por intenção de tratar, cujo significado para um único paciente é passível de críticas (22), tem a sua interpretação prejudicada, quando um grande número de pacientes deixa de receber o tratamento proposto.

Apesar da mortalidade associada do TMO alogênico ser maior, a expectativa é que ela seja reduzida nos estudos subsequentes devido a um melhor controle de complicações associadas à DECH e ao CMV.

\section{Transplantes não a parentados em LMA}

Cerca de 2/3 dos pacientes candidatos ao TMO alogênico não possuem um doador HLA 
idêntico. Apesar de existirem outras alternativas como 0 autotransplante e a quimioterapia intensiva, para a maioria dos pacientes com doença resistente, estas não representam uma solução curativa.

Os resultados iniciais com doadores não aparentados em LMA foram promissores porém com resultados inferiores aqueles observados com doadores HLA idênticos. Uma análise recente de 570 pacientes avaliados pelo NMDP demonstrou um possível resgate para cerca de $10 \%$ dos pacientes em fases avançadas da doença. Os resultados mais favoráveis em 1 a e $2^{2}$ a remissão indicam que a busca de um doador não aparentado deve ser considerada precocemente quando um doador familiar não for identificado na avaliação inicial destes pacientes (23).

\section{Recidivas Pós Transplante Alogênico}

As recidivas permanecem como um importante obstáculo ao sucesso do TMO e cerca de $50 \%$ dos pacientes com doença avançada demonstram doença recorrente. As respostas à quimioterapia nos primeiros 100 dias são limitadas, com cerca de $40 \%$ de óbitos após o transplante. Pacientes que apresentam uma recidiva após o primeiro ano de TMO apresentam uma evolução mais favorável. Alguns pacientes que entram em remissão com o tratamento quimioterápico podem ser submetidos a um segundo transplante e cêrca de $30 \%$ podem apresentar uma sobrevida prolongada (24).

Embora os resultados mais favoráveis com infusão de linfócitos como tentativa de induzir remissão tenham sido descritos na LMC, 0 mesmo efeito também pode ser observado em pacientes portadores de LMA que apresentam uma recidiva pós TMO (25). Apesar das complicações frequentes como a DECH e pancitopenia, cerca de $20 \%$ dos pacientes apresentam respostas favoráveis com eliminação do clone leucêmico.

Os estudos com infusão de linfócitos demonstram a importância do efeito imunológico na capacidade do TMO em curar leucemias. Esta estratégia modificou a abordagem dos regimes de condicionamento que hoje começam a ser considerados como uma plataforma para se desenvolver tolerância às células do doador, responsáveis em última análise pela eliminação do clone leucêmico. Os chamados "mini-

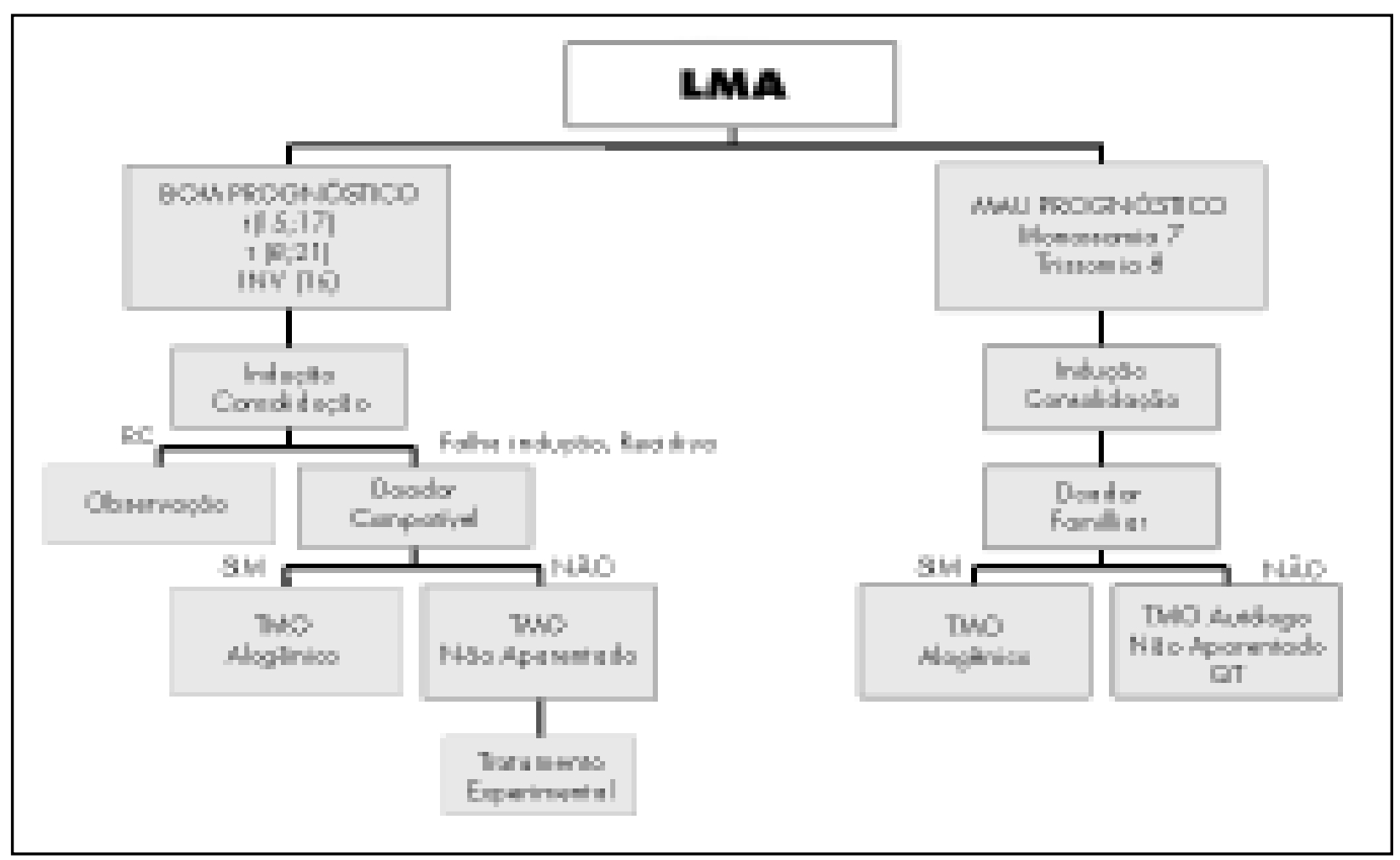

Quadro 1. Tratamento da LMA e o TMO 
transplantes" ou regimes de condicionamento não mieloablativos, constituem uma alternativa para pacientes mais idosos e que precisam ser submetidos a um transplante alogênico (26). Esta abordagem ainda deve ser considerada como um tratamento experimental.

\section{Conclusão}

Todos os pacientes com o diagnóstico de leucemia mielóide aguda devem ser submetidos a tipagem HLA. A abordagem inicial depende de avaliação citogenética, disponibilidade de um doador HLA idêntico e resposta a quimioterapia inicial. 0 tratamento pode ser orientado de acordo com o quadro 1 (2).

\section{Bone marrow transplantation in acute myelogenous leukemia}

Daniel G. Tabak

\begin{abstract}
Bone marrow transplantation represents an effectivetherapy for acute myelogen ousleukemia. Despite its initial use in patients in late stages of their disease, the best results have been documented when the procedure is undertaken while in first completeremission. Recentadvances in the management of the neutropenic patient have guaranteed the safe use of aggressive chemoterapy regimens, resulting in a prolonged disease free survival, similar to what is documented after myeloablativeregimens. Doubts still remain as of which patients should be submitted to marrow transplantation and at which stage of their disease. Cytogenetics and a better definition of other biological characteristics will allow a better selection of patients. The control of graft versus host disease, improvement in the management of infectious complications, the use of nonmyeloablative conditioning regimens and a greater availability of unrelated marrow donors will permit a greater use of allogeneic marrow transplantation in acute myelogen ous leukemia, including elderly patients. The role of autologous transplantation must be better defined.

Rev.bras.hematol.hemoter., 2000, 22(1):55-62

Key-words: acute myelogen ou leukemia, bone marrow transplantation treatment.
\end{abstract}

\section{Referências bibliográficas}

1. Thomas E.D., Buckner C.D., Banaji M., et al. One hundred patients with acute leukemia treated by chemotherapy, total body irradiation, and allogeneic marrow transplantation. Blood 1977; 49:511-33.

2. Stockerl-Goldstein K.E. and Blume K.G. Allogeneic hematopoietic cell transplantation for adult patients with acutemyeloid leukemia in Hematopoietic Cell Transplantation $2^{\text {nd }}$ Edition, Eds E Donnall Thomas; Karl G. Blume and Stephen J. Forman, BlackwellScience, $p p$ 823-834.

3. Mayer R.J., Davis R.B., Schiffer C.A., Berg $D . T$., et al. Intensive post remission chemotherapy in adults with acute myeloid leukemia. Cancer and Leukemia Group B. N Engl J Med 1994; 331: 896-903.

4. Byrd J. C., Dodge R. K., Andrew Carroll, et al. Patients With $t(8 ; 21)(q 22 ; q 22)$ and Acute Myeloid Leukemia Have Superior Failure-Free and Overall Survival When Repetitive Cycles of High-Dose Cytarabine Are Administered J Clin Oncol 1999 17: 3767-3775.

5. Appelbaum F.R. Allogeneic hematopoietic stem cell transplantation for acuteleukemia Sem. Oncol 1997; 24:114-123.

6. Tabak D.G., Bouzas L.F., Matta J.L., et al. Bone marrow transplantation in childhood acute myelogenousleukemia in first complete remission: Proceedings $\mathbf{1 7}^{\text {th }}$ International Cancer Congress, August 23 -28, Rio de Janeiro, Brazil, 1998.

7. Soiffer R.J., Fairclough D., Robertson M.,et al. CD6-depleted allogeneic bone marrow transplantation for acute leukemia in first complete remission. Blood. 1997; 15; 89:3039-47.

8. Locatelli F., Zecca M., Rondelli R., et al. Graft versus host disea se prophylaxis with low-dose cyclosporine-A reduces the risk of relapse in children with acute leukemia given HLAidentical sibling bone marrow transplantation: results of a randomized trial. Blood 2000; 95: 1572-9.

9. Blaise D., Maraninchi D., Archimbaud E., et al. Allogen eic bone marrow transplantation for acutemyeloid leukemia in firstremission: 
a randomized trial of a busulfan-Cytoxan versus Cytoxan-total body irradiation as preparative regimen: a report from the Group d'Etudes de la Greffe de Moelle Osseuse. Blood 1992; 79: 2578-82.

10. Clift R.A., Buckner C.D., Appelbaum F.R., et al. Long-Term Follow-Up of a Randomized Trial of Two Irradiation Regimens for Patients Receiving Allogeneic Marrow Transplants During First Remission of Acute Myeloid Leukemia. Blood 1998; 92: 1455-1456.

11. Ferrant A., Labopin M., Frassoni F., et al. Karyotype in acute myeloblastic leukemia: prognostic significance for bone marrow transplantation in first remission: a European Group for Blood and Marrow Transplantation study. Acute Leukemia Working Party of the European Group for Blood and Marrow Transplantation (EBMT). Blood. 1997; 90: 2931-8.

12. Gale R.P., Horowitz M.M., Rees J.K., et al. Chemotherapy versus transplants for acute myelogenous leukemia in second remission. Leukemia 1996; 10:13-9.

13. Clift R.A., Buckner C.D., Thomas E.D., et al. The treatment of acute nonlymphoblastic leukemia by allogeneic marrow transplantation. Bone Marrow Transplant. 1987; 2:243-58.

14. Grigg AP, Szer J, Beresford J, et al. Factors affecting the outcome of allogeneic bone marrow transplantation for adult patients with refractory or relapsed acute leukaemia. Br J Haematol. 1999; 107:409-18.

15. Brenner M.K., Rill D.R., Moen R.C., et al. Gene-marking to trace origin of relapse after autologous bone-marrow transplantation. Lancet. 1993; 341:85-6.

16. Vellenga E., van Putten W.L., Boogaerts M.A., et al. Peripheral blood stem cell transplantation as an alternative to autologous marrow transplantation in the treatment of acute myeloid leukemia? Bone Marrow Transplant. 1999; 23:1279-82.

17. Laporte J.P., Douay L., Lopez M., et al. One hundred twenty-fiveadultpatients with primary acuteleukemia autografted with marrow purged by mafosfamide: a 10-year single institution experience. Blood. 1994; 84:3810-8.
18.Gorin N.C.. Autologous stem cell transplantation in acute myelocytic leukemia. Blood. 1998; 92: 1073-90.

19. Bordignon C., Carlo-Stella C., Colombo M.P., et al. Cell therapy: achievements and perspectives. Haematologica. 1999; 84: 1110-49.

20. Gray R., Wheatley K. How to avoid bias when comparing bone marrow transplantation with chemotherapy. Bone Marrow Transplant 1991; 7 Suppl 3:9-12.

21. Burnett A.K., Kell J.. High dose cytarabine or transplantation for consolidation of younger patients with acute myeloid leukemia Curr Opin Oncol 2000; 12:110-115.

22. Frassoni F. Randomised studies in acute myeloid leukaemia: the double truth. Bone Marrow Transplant. 2000; 25:471-2.

23. Anasetti C, Petersdorf EW, Martin PJ et al. Improving availability and safety of unrelated donor transplants. Curr Opin Oncol 2000; 12:121-126.

24. Mehta J., Powles R., Treleaven J., et al. Outcome of acute leukemia relapsing after bonemarrow transplantation: utility of second transplants and adoptive immunotherapy. Bone Marrow Transplant 1997; 19:709-19.

25. Kolb H.J., Holler E. Adoptiveimmunotherapy with donor lymphocyte transfusions. Curr Opin Oncol. 1997; 9:139-45.

26. Champlin R., Khouri I., Kornblau S., et al. Allogeneic hematopoietic transplantation as adoptive immunotherapy. Induction of graft-versus-malignancy as primary therapy. Hematol Oncol Clin North Am. 1999; 13:1041-57.

Recebido: $05 / 03 / 2000$

Aceito: $24 / 03 / 2000$

\section{Siglas e instituições citadas}

CALGB: Cancer and Leukemia Group B

IBMTR: International Bone Marrow Transplant Registry

EORTC: European Organization for Research and Treatment of Cancer GIMEMA: Gruppo Italiano Malatie Ematologiche Dell'Adulto ECOG: Eastern Cooperative Oncology Group SMOG: South Western Cooperative Oncology Group GOELAM: Group d'Etudes de la Grette de Moelle Osseuse EBMT: European Group for Blood and Bone Marrow Transplantation 